\title{
Grupos empresariales y reportes de sostenibilidad
}

\section{Business groups and sustainability reports}

1 Julio C. Cantillo Padrón.

Universidad de Sucre (UNISUCRE), Sincelejo, Colombia

https://orcid.org/0000-0001-7589-7877

jccantillop@unisucrevirtual.edu.co

2 Aminta I De La Hoz Suárez.

https://orcid.org/0000-0001-6230-8869

Universidad de Sucre (UNISUCRE), Sincelejo, Colombia.

aminta.delahoz@unisucrevirtual.edu.co

3 Aris Ramírez Junieles.

(iD) https://orcid.org/0000-0001-7314-1267

Universidad de Sucre (UNISUCRE), Sincelejo, Colombia

aris.ramirez@unisucre.edu.co

Artículo de Investigación Científica y Tecnológica

Enviado: 12/12/2021

Revisado: $27 / 12 / 2021$

Aceptado: 28/01/2022

Publicado:05/02/2022

DOI: https://doi.org/10.33262/ap.v4i1.1.140

Cantillo Padrón, J. C., De La Hoz Suárez, A. I., \& Ramírez Junieles, A. (2022). Grupos empresariales y reportes de sostenibilidad . AlfaPublicaciones, 4(1.1), 56-64. https://doi.org/10.33262/ap.v4i1.1.140

ALFA PUBLICACIONES, es una Revista Multidisciplinar, Trimestral, que se publicará en soporte electrónico tiene como misión contribuir a la formación de profesionales competentes con visión humanística y crítica que sean capaces de exponer sus resultados investigativos y científicos en la misma medida que se promueva mediante su intervención cambios positivos en la sociedad. https://alfapublicaciones.com

Ciencia La revista es editada por la Editorial Ciencia Digital (Editorial de prestigio registrada en la Cámara Ecuatoriana

Digital

de Libro con No de Afiliación 663) www.celibro.org.ec

Esta revista está protegida bajo una licencia Creative Commons Attribution Non Commercial No Derivatives

4.0 International. Copia de la licencia: http://creativecommons.org/licenses/by-nc-nd/4.0/ 


\section{Palabras}

claves:

reportes de

sostenibilidad,

grupos

económicos, creación de valor, toma de decisiones, teoría de partes interesadas.

\section{Keywords:}

sustainability reports, economic groups, value creation, decision making, stakeholder theory.

\section{Resumen}

Este documento tiene como objetivo analizar las prácticas presentes en los informes de sostenibilidad y las estrategias de comunicación implementadas en 4 grupos económicos en Colombia. Para lograr este objetivo se tomó como referencia la teoría de las partes interesada y los planteamientos realizados por (Correa, 2018). En el desarrollo de la investigación se realizó un análisis de contenido en los informes de sostenibilidad presentados durante los años 2017 hasta el año 2019. Se logra concluir que los informes promueven una comunicación fluida con algunas de las partes interesadas, en términos de la toma de decisiones, gestión sostenible y creación de valor compartido; se evidencia un marcado énfasis en dos actores (los trabajadores y los inversionistas) y no hay consenso en la forma de revelar la información entre grupos económicos.

\begin{abstract}
This document aims to analyze the practices present in sustainability reports and communication strategies implemented in 4 economic groups in Colombia. To achieve this objective, the theory of the interested parties and the approaches made by Correa (2018) were taken as a reference. In the development of the research, a content analysis was carried out in the sustainability reports presented during the years 2017 to 2019. It is concluded that the reports promote fluid communication with some of the stakeholders, in terms of decision-making, sustainable management and creation of shared value; there is a marked emphasis on two actors (workers and investors) and there is no consensus on how to disclose information between economic groups.
\end{abstract}

\section{Introducción}

La incertidumbre y complejidad política, económica, financiera y social en los países han generados grandes desafíos en el sector empresarial. Para dar respuesta a estos desafíos, las organizaciones en Colombia han desarrollado estrategias de cooperación prolongada, impulsando de esta forma la creación de grupos empresariales. Estos grupos tiene como objetivo, entre otros aspectos, la generación de crecimiento sostenible, diversificación de las actividades, disminución de riesgo inherentes a las actividades económicas y la 
reducción de costos de operación, es decir, generar mayor valor empresarial, principalmente para los socios e inversionistas.

Producto de estas estrategias de cooperación durante décadas los grupos empresariales han "acumulado un poder económico considerable que obstaculiza la competencia y ejerce una influencia considerable en la política y en la regulación de los países" (Hamdani et al., 2020; Nathaniel, 1978). En tal sentido, los autores Kosenko et al. (2020) señalan que los grupos empresariales, en muchas economías emergentes, generan valor empresarial por los "vacíos institucionales, mercados financieros, laborales o de bienes intermedios subdesarrollados, sistemas judiciales ineficientes o corruptos, o falta de mecanismos de apoyo a la innovación" p. 4. Es decir, que los grupos empresariales generan mayor valor empresarial por el dominio que tienen sobre los activos productivos de un país. A partir de lo anteriormente planteado y teniendo en cuenta que los estados financieros contables tradicionales no reflejan la acciones en beneficio de los entornos social, es necesario analizar ¿Cómo los grupos empresariales en Colombia reflejan la creación de valor en sus reportes de sostenibilidad?

Para dar respuesta a este interrogante en el documento se analiza diferentes definiciones del concepto de grupo empresarial, se describe como se generó el surgimiento de los reportes de sostenibilidad y se presentan los hallazgos generados por el análisis de contenido en los informes de sostenibilidad presentados por cuatro grupos económicos colombianos durante los años 2017 hasta el año 2019. Esta investigación se centra en los grupos empresariales y busca presentar un análisis diferente en términos del énfasis, dado que las investigaciones normalmente centran su atención en la asociación entre las prácticas de sostenibilidad y el desempeño financiero de las empresas, es decir, en la importante en la mejora del desempeño financiero (Alshehhi et al., 2018).

\section{Metodología}

\section{Grupos empresariales en Colombia}

Antes de abordar el planteamiento del problema es necesario definir ¿Que es un grupo empresarial? Este puede ser definido como "un mecanismo de asignación jerárquica de recursos superando vacíos institucionales y permitiendo coordinar desarrollo de múltiples industrias interdependientes”. El autor Rodríguez (2021) señala que un grupo empresarial "es una colección de empresas legalmente independientes que están vinculadas por múltiples vínculos, incluida la propiedad, los valores económicos y las relaciones sociales (familia, parentesco, amistad), a través de las cuales se coordinan para lograr objetivos mutuos" (p. 6).

Según la OECD (2015) un grupo empresarial se define como un conjunto de empresas en las que existe una relación de subordinación con respecto a la misma explotación 
empresa, así como un acuerdo de subordinación con una "unidad de propósito" y dirección". En Colombia, existe una diferencia regulatoria entre "control" situaciones" y "grupos empresariales". En tal sentido, los grupos empresariales están conformados por vínculos entre las empresas y normalmente coordinan de acciones colectivas en beneficio de quienes ejercen control o la gestionan.

En contextos de económicas emergente los grupos empresariales generalmente se forman para protegerse de otras grupos o empresas competidores, para generar ventajas fiscales, ser más eficientes en sus procesos y generar economías de escalas. La forma más eficiente para el crecimiento de un grupo empresarial es realizando combinación de negocios. Según los estándares internacionales de Contabilidad una combinación de negocio implica, la compra de una parte de una empresa, de los títulos que representen la propiedad de otra, o bien la adquisición de los activos netos de la otra compañía, es decir, se genera mayor crecimiento adquiriendo negocios con actividades económicas similares o complementarias, a este tipo de estrategias son conocida como combinación de negocio horizontal o vertical.

Desde el punto de vista normativo para ser considerado un grupo empresarial en Colombia, se debe analizar la existencia del control, es decir, una o varias empresas del grupo deben estar controlado por otra empresa. Existen tres presunciones en las que una empresa debe estar considerado controlado: primero, tener más del 50\% de su propiedad pertenece a una sociedad holding, directamente o a través de otras filiales; segundo control de una sociedad holding y sus filiales en la junta de accionistas, o tener derecho a elegir a la mayoría de la junta directiva; y en tercera instancia, si una sociedad de cartera o sus filiales ejercen control o influencia significativa sobre el proceso de toma de decisiones de la empresa.

Dentro de las obligaciones normativas de un grupo empresarial en Colombia se encuentran: "presentar de un informe especial a inversionistas que indique las relaciones económicas existentes entre la controlantes, filiales o subsidiarias, inscripción en el registro mercantil, pago de dividendos y la elaboración y presentación de estados financieros consolidados" (Waked, 2018, p. 23).

De igual manera, el orden jurídico señala una serie de limitaciones "prácticas restrictivas o conductas censurables como: acordar precios, repartirse mercados, fijar cuotas de producción, menor calidad de bienes, determinar condiciones de venta, impedir a terceros entrar en el mercado, limitar desarrollos técnicos, disminución de precios, influenciar negativamente a la competencia" (Waked, 2018, p. 23).

La importancia de los grupos empresariales radica en la generación de empleo, pago de impuestos y generación de mayor producto interno bruto en los países. Según datos de la OECD (2015) la bolsa de valores de Colombi: "cuenta con 40 sociedades cotizadas de 77 
que forman parte de un grupo empresarial, 28 de ellos son sociedades holding y 12 subsidiarias, representando más del $60 \%$ de las acciones que son cotizan entre empresas que forman el índice COLCAP” (p. 69). El índice COLCAP refleja las variaciones de los precios de las acciones más importante de la Bolsa de Valores de Colombia (BVC) por lo cual se puede establecer una gran concentración de recursos en pocos grupos empresariales.

\section{Resultados y Discusión}

Los rápidos cambios generados por los avances tecnológicos, la globalización y la presión sobre las organizaciones para cambiar su modo de producción a uno menos perjudicial para el medio ambiente y la sociedad han impulsado la presentación de informes de sostenibilidad (Muhammad et al., 2020). El surgimiento de estos informes ha incrementado el interés de la comunidad académica y en especial en algunos académicos e instituciones de estandarización no estatal, como el American Institute of Certified Public Accountants (AICPA), Global Reporting Initiative (GRI), el Proyecto de Contabilidad Sostenible (A4S) y la International Federation of Accountants (IFAC) para que los informes contables tradicionales se integren mejor a las necesidades sociales (Haller \& Van Staden, 2014), dado que los informes de sostenibilidad permiten comunicar las contribuciones realizadas por las organizaciones a la sociedad, el medio ambiente y la generación de valor a sus partes interesadas.

Como se ilustra en la tabla 1 los informes de sostenibilidad se han transformado con el pasar del tiempo. Los contenidos inicialmente tenían un enfoque financiero, sin embargo, se han incluido indicadores de desempeño no financiero. Es de vital importancia resaltar que desde sus inicios y hasta la actualidad la presentación de este tipo de informes son iniciativas de carácter voluntario en Colombia.

\section{Tabla 1}

Evolución del reporting sobre información financiera y no financiera

\begin{tabular}{lll}
\hline \multicolumn{1}{c}{ Cronología } & \multicolumn{1}{c}{ Tipo de informe } & \multicolumn{1}{c}{$\begin{array}{c}\text { Dimensiones incluidas en el } \\
\text { contenido de los informes }\end{array}$} \\
\hline Antes de los 70 & Informes con contenido financiero. & $\begin{array}{l}\text { Dimensión exclusivamente } \\
\text { financiera. }\end{array}$ \\
Década de los años 70 y & Informes aislados de información & $\begin{array}{l}\text { Una dimensión financiera aislada } \\
\text { de la información social y }\end{array}$ \\
& financiera. & ambiental. \\
& Informes aislados de información & Una dimensión de sostenibilidad \\
& social o ambiental. & (social o ambiental). \\
\hline
\end{tabular}


Tabla 1

Evolución del reporting sobre información financiera y no financiera

\begin{tabular}{|c|c|c|}
\hline Cronología & Tipo de informe & $\begin{array}{c}\text { Dimensiones incluidas en el } \\
\text { contenido de los informes }\end{array}$ \\
\hline Década de los 90 & $\begin{array}{l}\text { Informes aislados de información } \\
\text { financiera. } \\
\text { Informes especializados en } \\
\text { sostenibilidad que conjugan } \\
\text { información social ambiental. }\end{array}$ & $\begin{array}{l}\text { Dimensión financiera } \\
\text { Dos dimensiones de sostenibilidad } \\
\text { que ganan vigor: ecológica y social }\end{array}$ \\
\hline Década del 2000 & $\begin{array}{l}\text { Algunas iniciativas de combinar } \\
\text { información de sostenibilidad e } \\
\text { información financiera bajo el } \\
\text { Informe anual. }\end{array}$ & $\begin{array}{l}\text { Recuperación de la importancia de } \\
\text { la dimensión financiera de los } \\
\text { Informes y auge de la información } \\
\text { con contenido no financiero. }\end{array}$ \\
\hline Tendencia actual & $\begin{array}{l}\text { Un único Informe Integrado (visión } \\
\text { holística de la organización). }\end{array}$ & $\begin{array}{l}\text { Tres dimensiones de sostenibilidad } \\
\text { (financiera, ecológica y social en } \\
\text { equilibrio). }\end{array}$ \\
\hline
\end{tabular}

Fuente: Barandica \& Díaz (2018), Rivera et al. (2016)

\section{Conclusiones}

- Según el estudio realizado por Correa (2018), se establecen tres estrategias de comunicación entre los grupos empresariales y sus partes interesadas, la primera se refiere a un tipo de estrategia denominada como "unidireccional simétrica", esta solo tienen un carácter divulgativo; la segunda conocida como "estrategia de respuesta", en la que existe un flujo de comunicación de doble vía y en la tercera, denominada como "participación” se plantean mecanismo de retroalimentación y de articulación permanente.

- A partir del análisis de contenido en los informes de sostenibilidad presentados durante los años 2017 hasta el año 2019 se pudo establecer un marcado énfasis en generar valor en dos actores los trabajadores e inversionistas, a pesar de promover una comunicación fluida con algunas de las partes interesadas, en términos de la toma de decisiones, gestión sostenible y creación de valor compartido; se evidencia un marcado énfasis en dos actores. A pesar de implementar estrategias de interacción y socialización de acciones se resaltan los impactos en términos de generación de bienestar de los trabajadores y los inversionistas, excluyéndose el resto e interesados como: proveedores, acreedores, Clientes, estado y en general la comunidad que conforma el entorno cercano a las organizaciones.

- De igual forma, se evidencia una tradición voluntaria en términos de divulgación y acciones específicas, lo cual genera asimetría en los informes; por no existir un marco normativo que regule el tema y no tener una guía que permita generar homogenizar o estandariza la forma de revelar la información de los grupos económicos. De igual forma, es necesario avanzar en los mecanismos que promuevan en mayor medida la participación de los stakeholders en la toma de 
decisiones relacionadas con la gestión sostenible y la creación de valor compartido (Correa, 2018).

\section{Referencias Bibliográficas}

Alshehhi, A., Nobanee, H., \& Khare, N. (2018). The impact of sustainability practices on corporate financial performance: Literature trends and future research potential. Sustainability (Switzerland), 10(2). https://doi.org/10.3390/su10020494

Barandica, J., \& Díaz, M. (2018). Origen y evolución de los reportes integrados. Gerencia Libre, 5, 79-89.

Correa, J. (2018). Grupos Empresariales y Reportes de Sostenibilidad en América Latina. https://roderic.uv.es/handle/10550/67806

Haller, A., \& Van Staden, C. (2014). The value-added statement - an appropriate instrument for integrated reporting. Accounting, Auditing and Accountability Journal, 27(7), 1190-1216. https://doi.org/10.1108/AAAJ-04-2013-1307

Hamdani, A., Kosenko, K., \& Yafeh, Y. (2020). Regulatory Measures to Dismantle Pyramidal Business Groups: Evidence from the United States, Japan, Korea and Israel.

Kosenko, K., Yafeh, Y., \& Hamdani, A. (2020). Regulatory Measures to Dismantle Pyramidal Business Groups: Evidence from the United Regulatory Measures to Dismantle Pyramidal Business Groups: Evidence from the United. Journal of Law, Finance, and Accounting, 6(2), 1-43.

Muhammad K., Lai, F., Shamim, A., \& McShane, M. (2020). The efficacy of sustainability reporting towards cost of debt and equity reduction. Environmental Science and Pollution Research, 27(18), 22511-22522. https://doi.org/10.1007/s11356-020-08398-9

Nathaniel, L. (1978). Industrial Organization and Entrepreneurship in the Developing Countries: The Economic Groups. Economic Development and Cultural Change, 26(4), 661-675. https://doi.org/10.1086/451052International Accounting Standards Board., 2018 Fundación IFRS 149 (2018).

OECD. (2015). Gobierno Corporativo de Grupos Empresariales en América Latina. 
Rivera, Y., Zorio, A., \& García, M. (2016). The integrated reporting concept as an innovation in corporate reporting. Journal of Innovation and Knowledge, 1(3), 144-155. https://doi.org/10.1016/j.jik.2016.01.016

Rodriguez, A. (2021). Assessing the impact of corporate entrepreneurship in the financial performance of subsidiaries of Colombian business groups_ under environmental dynamism moderation _ Enhanced Reader.pdf (pp. 1-27).

Waked, R. (2018). Nociones de los grupos empresariales por sus alcances en la ley Notions of business groups for their scope in Colombian law.

\section{LCiencia}


El artículo que se publica es de exclusiva responsabilidad de los autores y no necesariamente reflejan el pensamiento de la Revista Alfa Publicaciones.

\section{Ciencia}

El artículo queda en propiedad de la revista y, por tanto, su publicación parcial y/o total en otro medio tiene que ser autorizado por el director de la Revista Alfa Publicaciones.
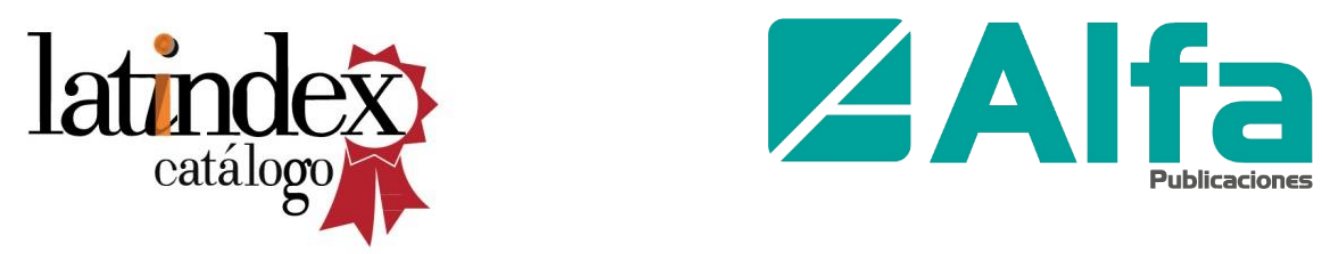

doi crosiret latindex ILatinREV
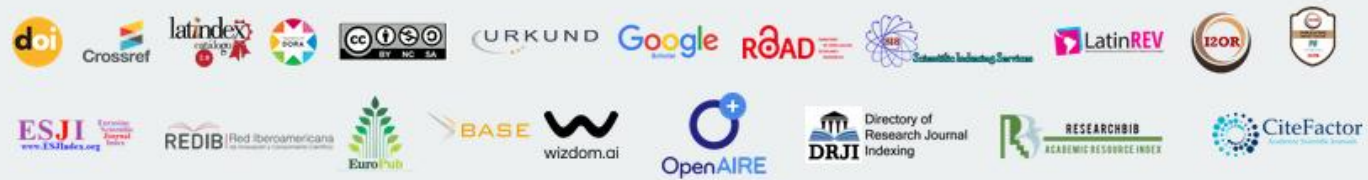